BMJ Open

Diabetes

Research

\& Care

\title{
Increased risk of hospital-acquired foot ulcers in people with diabetes: large prospective study and implications for practice
}

\author{
Frances Wensley, ${ }^{1}$ Christopher Kerry, ${ }^{2}$ Gerry Rayman ${ }^{2}$
}

To cite: Wensley F, Kerry C, Rayman G. Increased risk of hospital-acquired foot ulcers in people with diabetes: large prospective study and implications for practice. BMJ Open Diab Res Care 2018;6:e000510. doi:10.1136/ bmjdrc-2018-000510

- Additional material is published online only. To view please visit the journal online (http://dx.doi.org/10.1136/ bmjdrc-2018-000510)

Received 16 January 2018 Accepted 28 April 2018

Check for updates

${ }^{1}$ Royal Free Hospital NHS Trust, London, UK

${ }^{2}$ Ipswich Hospital NHS Trust, Ipswich, UK

Correspondence to Professor Gerry Rayman; gerry.rayman@ipswichhospital. nhs.uk

\section{ABSTRACT}

Aims Diabetes increases the risk of costly and potentially preventable hospital-acquired pressure ulceration. Given that peripheral arterial disease and neuropathy, important risk factors for foot ulceration, are more common in people with diabetes, their risk of hospital-acquired foot ulceration (HAFU) in particular may be even greater. This study aims to determine this risk.

Methods Using data collected over 2 years from all admissions to the Ipswich Hospital NHS Trust, we conducted a prospective multilevel regression analysis of the risk of HAFU in 5043 admissions of people with diabetes versus 23599 without diabetes. Patients over 50 years who developed HAFU at least 48 hours after admission were included in analyses. Progressive adjustment for important risk factors and subgroup analyses were conducted to compare patients with and without diabetes.

Results There were significant differences between patients with and without diabetes among a range of covariates including sex, Comorbidity Score, and length of stay ( $p$ value $<0.001$ ). After progressive adjustment for age, sex, and other risk factors, there persisted a significant increase risk of HAFU in people with diabetes (OR 2.24; 95\% Cl 1.80 to 2.69). There were no substantial differences between clinically relevant subgroups. Conclusions These analyses demonstrate at least a twofold increase in the risk of HAFU in patients with diabetes and suggest further work should focus on specific processes to detect those inpatients with diabetes at increased risk, in whom preventative measures may reduce the prevalence of this costly complication.

\section{INTRODUCTION}

Hospital-acquired pressure ulcers are associated with significant morbidity and mortality and are a major financial burden to global healthcare systems. They are estimated to cost 11 billion dollars annually in the USA and 2.6 billion pounds annually in the UK, not including litigation and societal costs. ${ }^{12}$ Accurately identifying those at risk is important for prevention. Risk factors include immobility, nutritional deficiency, cognitive impairment, history of previous pressure ulceration, impaired circulation, and sensory loss. ${ }^{3-5}$
Diabetes has been shown to be an important risk factor and in a recent meta-analysis the pooled OR of the incidence of pressure ulcers in patients with diabetes was 1.74 (95\% CI 1.40 to 2.15 ) compared with patients without diabetes. ${ }^{6}$ Another recent meta-analysis found a slightly lower but significantly adjusted relative risk ratio of 1.17 (95\% CI 1.02 to 1.36) after sensitivity analysis accounting for publication bias. ${ }^{7}$ Of note nearly all reports to date, as well as those included in these meta-analyses, relate to pressure ulcers in surgical patients and at all anatomical sites combined, not specifically the foot. Since peripheral arterial disease and neuropathy are more common in people with diabetes, affecting approximately $30 \%$ and $40 \%-60 \%$ of patients respectively, it can be reasonably assumed that the risk of hospital-acquired foot ulceration (HAFU) may be of greater relevance in people with diabetes. ${ }^{8} 9$ To date however there have been no studies to quantify this risk. The UK National Diabetes Inpatient Audit (NaDIA in 2010) found that $2.2 \%$ of inpatients with diabetes developed a foot ulcer during their admission but data on the population without diabetes were not collected. ${ }^{10}$

The high cost, morbidity and mortality associated with hospital-acquired pressure ulcers has focused healthcare services on prevention and early treatment; guidance on this was recently issued by the National Institute for Health and Care Excellence in 2014 (clinical guidance (CG) 179) and the American College of Physicians in 2015..$^{11}$ Diabetes receives little mention as a risk factor in these comprehensive documents, despite accounting for approximately 15\%-30\% of the inpatient population. ${ }^{10} 13$ Furthermore, there is no mention of their greater vulnerability to foot ulceration. This may be due in part to the current paucity of large, 


\section{Significance of this study}

What is already known about this subject?

- Diabetes is associated with an increased risk of foot ulceration due to peripheral arterial disease and neuropathy. Foot ulceration can cause substantial morbidity and its complications have a significant impact on patients in terms of stay in hospital, recovery period and long-term mobility, with significant costs to healthcare systems. Previous small studies have looked at skin pressure ulceration in a range of inpatient populations, including in people with diabetes, and a recent meta-analysis demonstrated an increased risk of pressure ulcer at all skin sites combined in patients with diabetes versus those without. While there have been improvements in recognition and care of patients at risk of pressure ulcers over the past 10 years, in many parts of the UK and globally the prevalence of hospital-acquired pressure ulcers remains the same. In addition, with the increasing prevalence of diabetes, this potentially life-changing harm will assume an increasing importance.

What are the new findings?

- The current study, comprising over 5000 inpatients with diabetes, shows that diabetes is an important risk factor for hospital-acquired foot ulceration (HAFU) in particular, and that this association persists after adjustment for potential confounders and among clinically relevant subgroups.

How might these results change the focus of research or clinical practice?

By highlighting the strength of association between people with diabetes for HAFU, we hope to improve awareness of this risk among all healthcare professionals. Changes to clinical practice may include improved assessment of the diabetic foot on admission to hospital, inclusion of diabetes as a risk factor in scores for hospital-acquired pressure ulcers and improved management of patients to prevent heel ulceration. This study also indicates the need for further research to focus on developing specific processes to detect those inpatients with diabetes at greatest risk of HAFU and whether preventative measures focused on this group are effective in reducing this harm.

prospective studies to quantify the role of diabetes as a risk factor.

If people with diabetes are at increased risk of HAFU there could be a strong case for specific processes to detect those inpatients with diabetes at greatest risk, to whom preventative measures could be focused. The aim of this study was to determine whether the diagnosis of diabetes is associated with an increased risk of HAFU when compared with those without diabetes.

\section{RESEARCH DESIGN AND METHODS}

From early 2008, it became mandatory in our hospital to report all grade 2, grade 3 and grade 4 hospital-acquired pressure ulcers and their anatomical site to the Clinical Quality and Patient Safety Directorate using the Datix incident reporting system. ${ }^{14}$ Furthermore, a process was put in place for the tissue viability nursing team to continuously audit and report such events. Using their database, which includes all hospital-acquired pressure ulcers, the numbers of HAFU in a 2-year period, from 01 October 2008 to 30 September 2010, were isolated. Foot ulcers identified within 48 hours of admission were excluded to avoid inclusion of pressure ulcers acquired in the community. The number of patients who developed foot ulcers rather than total number of ulcers was counted, that is, bilateral ulcers were considered one patient event. Since the database did not always distinguish between those with and without diabetes, for all patients with a hospital-acquired foot lesion, the patient administration system (PAS), clinical case notes and electronic discharge letters were reviewed to identify all those with a diagnosis of diabetes. Additionally, for all patients with a reported HAFU, the chemical pathology database was searched to confirm the diagnosis in those reported to have diabetes and to identify those not recorded in the notes as having diabetes, and/or those missed by hospital coding. Any missing data points were sought through PAS. This database contains results of all blood tests obtained during the inpatient episode as well as any obtained in primary care over the previous 8 years and subsequent 2 years. The diagnosis of diabetes was made in accordance with the American Diabetes Association 2015 criteria. ${ }^{15}$ The total number of hospitalized patients with and without a diagnosis of diabetes and their length of stay during the 2-year period were identified through PAS.

This study forms part of the hospital's service improvement.

\section{Statistical analyses}

Data were summarized using means and SD or percentages of categorical variables. Admissions with data missing on diabetes, HAFU or any covariates were reviewed and missing data points were updated using PAS. Patients with type 1 and type 2 diabetes were considered together. Day admission patients (length of stay=1) were also excluded from analyses due to their very small likelihood of obtaining a pressure ulcer secondary to their hospital admission. Patients were followed up for the duration of their hospital stay. Variations between people with and without diabetes were estimated using two-sampled t-tests or rank sum tests (non-parametrical data) for difference between the means, $\chi^{2}$ tests for correlation between binary variables and analysis of variance (ANOVA) tests for comparison of categorical variables. To measure the effect of the diagnosis of diabetes on HAFU, two-level logistic models were estimated, whereby admissions were nested within patients. ${ }^{16}$ Models were progressively adjusted for potential confounding risk factors, including age, sex, length of stay, Charlson Comorbidity Score, ${ }^{17}$ day of admission, admission type and specialty. Further logistic regression analyses were conducted to visualize any difference between clinically relevant subgroups. Data were analysed using Stata V.12.1.

\section{RESULTS}

Data were collected on 18946 adult patients aged 50 years or older comprising all 28642 hospital admissions 
Table 1 Summary of data collected between October 2008 and September 2010

\begin{tabular}{|c|c|c|c|c|c|}
\hline \multirow[b]{2}{*}{ Variable } & \multicolumn{2}{|c|}{ Inpatients without diabetes } & \multicolumn{2}{|c|}{ Inpatients with diabetes } & \multirow[b]{2}{*}{ P value* } \\
\hline & $\mathbf{N}$ & $\begin{array}{l}\text { Mean (SD) or } \\
\mathrm{N}(\%)\end{array}$ & $\mathbf{N}$ & $\begin{array}{l}\text { Mean (SD) or } \\
\text { N (\%) }\end{array}$ & \\
\hline Age & 23599 & 74.70 (11.82) & 5043 & 75.25 (10.35) & 0.002 \\
\hline $\begin{array}{l}\text { Charlson } \\
\text { Comorbidity Score }\end{array}$ & 23599 & $2.27(2.39)$ & 5043 & $3.01(2.49)$ & $<0.001$ \\
\hline Length of stay & 23599 & $6.0(2.0$ to 33.0$) \dagger$ & 5043 & $7.0(2.0$ to 39.0$) \dagger$ & $<0.001$ \\
\hline Sex (\% male) & 23599 & 10719 (45.4) & 5043 & 2631 (52.2) & $<0.001$ \\
\hline Admission type & 23599 & & 5043 & & $<0.001$ \\
\hline Elective & & $5881(24.9)$ & & $784(15.5)$ & \\
\hline Non-elective & & 17718 (75.1) & & $4259(84.5)$ & \\
\hline Specialty & 23599 & & 5043 & & $<0.001$ \\
\hline Surgical & & $8710(36.9)$ & & $1351(26.8)$ & \\
\hline Medical & & 14889 (63.1) & & 3692 (73.2) & \\
\hline Day of admission & 23599 & & 5043 & & 0.336 \\
\hline Sunday & & 2637 (11.2) & & $592(11.7)$ & \\
\hline Monday & & 3906 (16.6) & & $831(16.5)$ & \\
\hline Tuesday & & 3897 (16.5) & & 780 (15.5) & \\
\hline Wednesday & & 3804 (16.1) & & 790 (15.6) & \\
\hline Thursday & & $3316(14.0)$ & & 745 (14.8) & \\
\hline Friday & & $3545(15.0)$ & & $750(14.9)$ & \\
\hline Saturday & & $2494(10.6)$ & & 555 (11.0) & \\
\hline \multicolumn{6}{|l|}{ Outcomes } \\
\hline Foot ulcer & 23599 & $119(0.50)$ & 5043 & $52(1.0)$ & $<0.001$ \\
\hline Mortality (\% died) & 23599 & $11065(47.0)$ & 5043 & $2769(54.9)$ & $<0.001$ \\
\hline
\end{tabular}

${ }^{*} \mathrm{P}$ value for difference between means ( $\mathrm{t}$-test), binary variables $\left(\chi^{2}\right.$ test) and categorical variables (analysis of varianceoVA). Rank-sum test used to compare non-parametrical variables.

†Median (IQR)

of at least 2 days between October 2008 and September 2010. Of these, $3076(16.2 \%)$ patients had a diagnosis of diabetes, comprising 5043 admissions. All admissions had data on diabetes, heel ulcer, length of stay and other covariates included in the analyses. Differences in baseline data between participants with and without diabetes are shown in table 1 . In general, patients with diabetes were slightly older (mean age 75.2 (SD 10.4) years for people with diabetes versus 74.7 (SD 11.8) years for people without diabetes, $\mathrm{p}$ value for $\mathrm{t}$-test $=0.002)$, more likely to be male $\left(52.2 \%\right.$ vs $45.4 \%$, $p$ value for $\chi^{2}$ test $\left.<0.001\right)$, had a slightly prolonged length of stay ( 7 days (IQR 2 days to 39 days) vs 6 days (IQR 2 days to 33 days), $\mathrm{p}$ value for rank-sum test $<0.001$ ) and had higher Charlson Comorbidity Scores (3.0 (SD 2.5) vs 2.3 (SD 2.4), p value for t-test $<0.001)$ compared with those without diabetes. One hundred and seventy-one $(0.60 \%)$ patients developed a heel ulcer during admission, including $52(1.0 \%)$ with and $119(0.50 \%)$ without diabetes ( $p$ value for $\chi^{2}<0.001$ ). People with diabetes were more likely to have emergency admissions ( $p$ value $<0.001$ ), and were more likely to be admitted under medical specialties than surgical
( $p$ value $<0.001$ ). There was no difference in the weekday of admission between groups ( $\mathrm{p}$ value for ANOVA test $=0.34$ ). Summary tables of diabetes and non-diabetes admissions by speciality and reason for admission are presented in the online supplementary file 1 .

Table 2 shows the association between diabetes and risk of HAFU. In the baseline model adjusted for age and sex, the OR for risk of HAFU in people with diabetics versus those without diabetes is 2.24 (95\% CI 1.87 to 2.62). This estimate persisted with progressive adjustment for major risk factors or confounders, including length of stay and comorbidities (OR 2.23; 95\% CI 1.79 to 2.67). Further adjustment for day of admission, medical and surgical specialty, or elective and emergency admission type did not have any bearing on the association. On further subgroup analyses there were no substantial differences in the association of diabetes and HAFU between men and women, elective and emergency admissions, or medical and surgical specialties (figure 1, p values for heterogeneity between subgroups $>0.05$ ). There was no significant difference in OR between days of admission (data not shown). 
Table 2 Association between diabetes and heel ulcer in 18946 patients with 28642 admissions between October 2008 and September 2010. The model was progressively adjusted for known and novel risk factors

\begin{tabular}{|c|c|c|c|}
\hline Model & OR $(95 \% \mathrm{Cl})$ & $\chi^{2}$ & $P$ value \\
\hline Adjusted for age and sex & 2.24 (1.87 to 2.62$)$ & 17.94243 & $<0.001$ \\
\hline Adjusted for above and length of stay & 2.21 (1.76 to 2.65$)$ & 12.33788 & $<0.001$ \\
\hline Adjusted for above and comorbidity (Charlson Index) & 2.23 (1.79 to 2.67$)$ & 12.61275 & $<0.001$ \\
\hline Adjusted for above and day of admission & 2.24 (1.80 to 2.69$)$ & 12.67092 & $<0.001$ \\
\hline Adjusted for above and specialty & 2.27 (1.82 to 2.72$)$ & 12.90961 & $<0.001$ \\
\hline Adjusted for above and admission type & 2.24 (1.79 to 2.69$)$ & 12.55047 & $<0.001$ \\
\hline
\end{tabular}

\section{DISCUSSION}

As far as we are aware this study comprises the largest prospective cohort of people with and without diabetes who have developed HAFU. It demonstrates that patients with diabetes are at least twice as likely to develop a foot ulcer during their hospital stay, irrespective of age, length of stay, reason for admission or comorbidities. Furthermore, this association did not vary among potentially relevant subgroups, including sex, type of admission or specialty.

There are a number of advantages of this study. First, the tissue viability nurses collected data prospectively across all admissions, and the clinical notes, PAS, and pathology databases were scrutinized for every patient with HAFU in order to identify and confirm diagnosis of diabetes. Furthermore, detailed data collection on important risk factors enabled adjustment for potential confounding factors. The availability of data on patient comorbidities (International Statistical Classification of Disease and Related Health Problems 10th revision
(ICD-10) codes) enabled more robust covariate analyses using the Charlson Index, a standardized comorbidity score.

As individual patients may have recurrent admissions during the study period, we analysed the data using a two-step regression approach to account for any biases associated with multiple admissions and thus multiple entries into the study. While this approach was used to improve the veracity of the results, the effect estimates did not differ markedly from straightforward single-step regression analyses.

Previous studies of pressure ulcers have looked at adult patients over the age of 16 years. However it is unusual for patients between the ages of 16 years and 49 years to develop HAFU even with prolonged hospital stay, and no one within this age group with diabetes in the study group developed HAFU during the data collection period. Furthermore, the Ipswich cohort comprised sufficiently high numbers of admissions to exclude these patients, leaving a large prospective data set of patients over the

\section{No. admissions \\ No. cases}

OR $(95 \% \mathrm{Cl})$

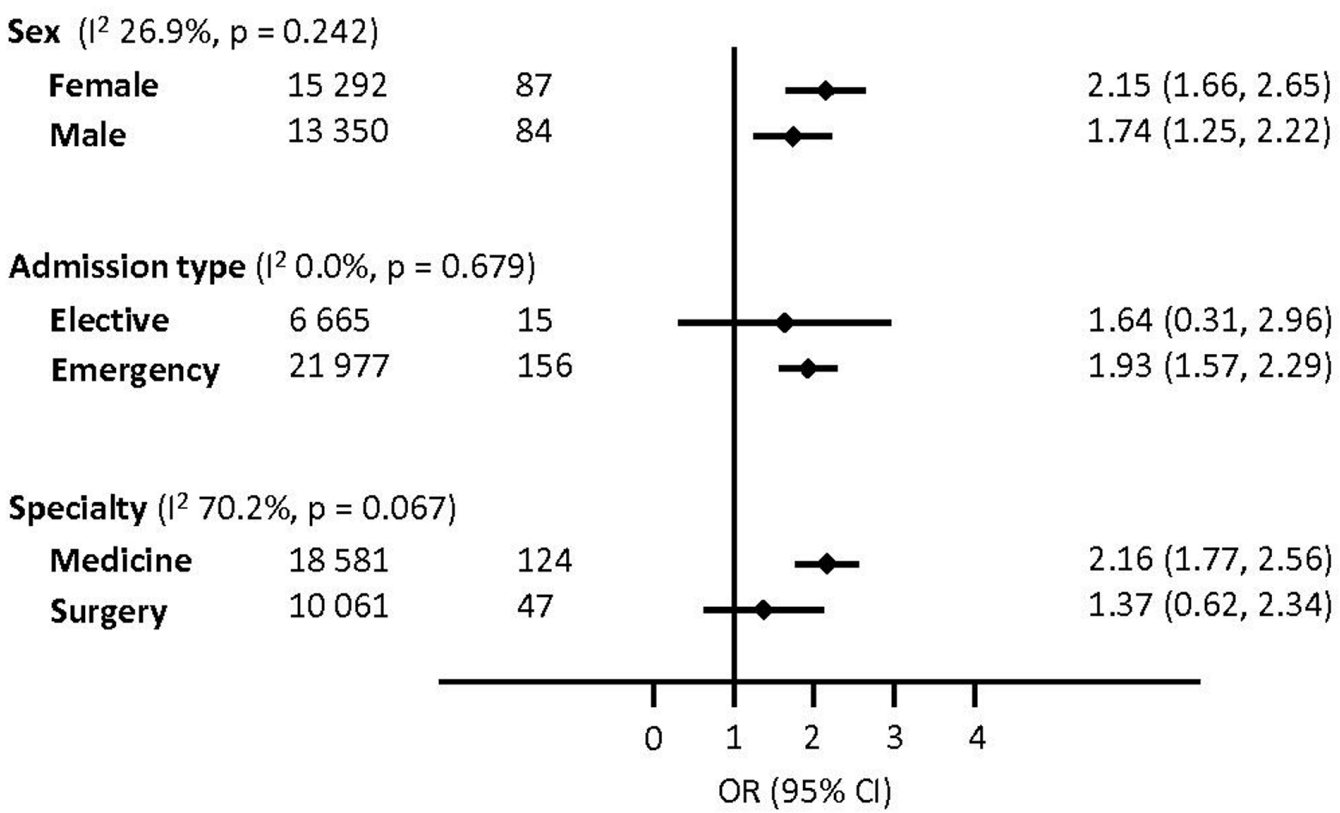

Figure 1 Association between diabetes and heel ulcer in selected subgroups. The model was adjusted for age, sex, length of stay and comorbidity (defined by the Charlson Comorbidity Index). 
age of 49 years. As patients with diabetes tend to be older than those without diabetes, excluding patients under 50 years greatly reduced the age-related variation between study groups; therefore the overall effect estimates were more precise.

Another advantage to the current analyses is the specificity of the outcome and its relevance to diabetes. Previous papers have looked at factors associated with increased risk of pressure ulcers at all sites in large cohorts of inpatients and have identified diabetes as a risk factor. As far as we are aware this is the first prospective study, which specifically addresses HAFU in people with diabetes. The only study of which we are aware that specifically aimed to identify risk factors associated with hospital-acquired heel pressure ulcers, found the OR risk for diabetes to be 2.9 (95\% CI 1.2 to 7.2$).{ }^{18}$ However, the authors highlighted the need for a prospective cohort study as their results were based on retrospective chart reviews. That the OR for our study is slightly lower (2.24) may be explained by the prospective study design. Additionally, our proactive multidisciplinary foot team, that has reduced amputation rates in our catchment area by over $75 \%$, could have prevented some HAFU through their inpatient activity. ${ }^{19}$ This is supported by the finding that only $1 \%$ of our inpatient population with diabetes had HAFU compared with 2.2\% in NaDIA 2010. ${ }^{10}$ Nevertheless, the current study shows that despite the presence of this team, HAFU in people with diabetes is at least twofold greater than in people without diabetes. The study also highlights the increased vulnerability of the foot compared with other anatomical sites, the relative risk being almost twice as great when compared with that reported in the previously described meta-analysis of all pressure ulcers (1.17). These findings suggest a specific need for interventions to protect the feet of patients with diabetes.

There are a number of limitations to this study. It is possible that some community-acquired foot ulcers were not identified or documented on admission and may therefore have been misclassified as HAFUs. We have attempted to mitigate this by excluding patients with a hospital stay less than 48 hours. It is also possible that some HAFUs were not identified prior to discharge. We believe that the numbers misclassified (not acquired in hospital or missed prior to discharge), if any, will be small as the tissue viability nurses who led the pressure ulcer prevention programme were specifically tasked and meticulous in collecting this data as part of the hospital's quality improvement programme; additionally it is mandatory to report all pressure ulcers via the DATIX reporting system. Furthermore, as data from both cohorts have been subjected to the same process, such errors, if any, will be common to both groups of patients. Another potential limitation is not including grade 1 ulcers. As our hospital only mandates reporting of ulcers of grade 2 and above, no data were available for grade 1 ulcers. Nevertheless, we see no reason why the increased risk should not also apply to grade 1 lesions. Finally, the data were collected from 2008 to 2010 . While there have been improvements in recognition and care of patients at risk of pressure ulcers over this time, in many parts of the UK and globally the prevalence of hospital-acquired pressure ulcers remains the same. In the UK, hospital-acquired pressure ulceration remains one of the four most common harms recorded in the NHS Safety Thermometer. ${ }^{20}{ }^{21}$ In addition, with the increasing prevalence of diabetes,${ }^{22}$ this potentially life-changing harm will assume increasing importance.

While the current study set out determine whether people with diabetes were at increased risk of HAFU, it should be recognized that the association in those with specific risk factors was not determined; that is, those who on admission were found to have neuropathy, peripheral arterial disease (absent foot pulses), known history of previous foot disease and significant renal impairment. It is likely that they are at even greater risk and identification and targeting these patients should be the priority. It was not possible to segregate our patients into those with and without risk factors, as these data were not always available in the notes. This is not unique to our hospital and indeed the UK NaDIA found that only a third of admissions have any foot risk assessment on admission. ${ }^{10}$ Again this highlights the importance of including all patients with diabetes in ward-based risk score assessments for pressure ulcers, until systems are in place which will ensure that all those with these risk factors are identified on admission.

The findings of this study are important for the care of people with diabetes in hospital. Hospital-acquired pressure ulceration is now recognized as a major burden and many countries have triggered nationwide prevention drives. In the UK reporting is mandatory and hospitals are benchmarked using the NHS Safety Thermometer. ${ }^{20}$ Diabetes as a risk factor however is not well publicized in these initiatives and as previously mentioned scarcely referred to in guideline documents. ${ }^{11}{ }^{12}$ Indeed, none of the common generic scoring systems for identifying those at risk, which include the Barlow, Braden, Norton and Waterlow systems, specifically includes diabetes as a risk factor. ${ }^{23-25}$ Furthermore, there is no scoring system to highlight the particular risk to the feet of people with diabetes. The 'Waterlow' Score, one of the most widely used, lists neurological deficit as a risk factor and mentions diabetes in this limited context. Since the 'Waterlow' Score is completed by ward nurses, who do not usually examine for neuropathy or peripheral vascular disease, patients at risk will be missed unless medical notes of those with diabetes are scrutinized to detect those with neuropathy or vascular disease; in practice this is infrequent. Furthermore, and of more significance, as previously mentioned only a third of people with diabetes have their feet risk assessed by medical staff at any time during their admission. ${ }^{10}$ Thus, the majority of people with diabetes who are likely to be at increased risk of HAFU are not highlighted for preventative treatment. 
In conclusion, this study confirms that people with diabetes have at least a twofold greater risk of HAFU than those without diabetes and highlights the need for all healthcare professionals to be aware that people with diabetes are at increased risk. It also indicates the need for further research to focus on developing specific processes to detect those inpatients with diabetes at greatest risk of HAFU and whether preventative measures focused on this group are effective in reducing this harm. This is particularly important as people with diabetic foot ulceration are at increased risk of progression to more serious complications including cellulitis, abscess formation, tissue necrosis, osteomyelitis, septicaemia, amputation and death. Further research will be needed to determine the effectiveness of current and future preventative interventions.

Acknowledgements The authors thank the Ipswich Hospital tissue viability team, the diabetes inpatients specialist team and the information technology team.

Contributors GR and CK designed the study and collected data. FW collated and analysed the data. FW and GR interpreted analyses and drafted the manuscript. All three authors had final approval of the version to be published.

Funding The Diabetes Centre Research Funds.

Competing interests None declared.

Patient consent Not required.

Ethics approval Ipswich Hospital Research and Audit Committee.

Provenance and peer review Not commissioned; externally peer reviewed.

Data sharing statement Data will be available on request to the corresponding author.

Open access This is an Open Access article distributed in accordance with the Creative Commons Attribution Non Commercial (CC BY-NC 4.0) license, which permits others to distribute, remix, adapt, build upon this work non-commercially, and license their derivative works on different terms, provided the original work is properly cited and the use is non-commercial. See: http://creativecommons.org/ licenses/by-nc/4.0/

(c) Article author(s) (or their employer(s) unless otherwise stated in the text of the article) 2018. All rights reserved. No commercial use is permitted unless otherwise expressly granted.

\section{REFERENCES}

1. Bennett G, Dealey C, Posnett J. The cost of pressure ulcers in the UK. Age Ageing 2004;33:230-5.

2. Russo CA, Steiner C, Spector W. Hospitalizations related to pressure ulcers among adults 18 years and older, 2006. Healthcare cost and urilization project (HCUP) Statistical Brief \#64. Washington, DC: Agency for Healthcare Research and Quality, 2008.

3. Frankel H, Sperry J, Kaplan L. Risk factors for pressure ulcer development in a best practice surgical intensive care unit. Am Surg 2007;73:1215-7.

4. Liu P, He W, Chen HL. Diabetes mellitus as a risk factor for surgery-related pressure ulcers: a meta-analysis. J Wound Ostomy Continence Nurs 2012;39:495-9.
5. Coleman S, Gorecki C, Nelson EA, et al. Patient risk factors for pressure ulcer development: systematic review. Int J Nurs Stud 2013;50:974-1003.

6. Kang ZQ, Zhai XJ. The Association between pre-existing diabetes mellitus and pressure ulcers in patients following surgery: a metaanalysis. Sci Rep 2015;115:13007.

7. Liang $\mathrm{M}, \mathrm{Chen} \mathrm{Q}$, Zhang $\mathrm{Y}$, et al. Impact of diabetes on the risk of bedsore in patients undergoing surgery: an updated quantitative analysis of cohort studies. Oncotarget 2017;8:14516-24.

8. Beks PJ, Mackaay AJ, de Neeling JN, et al. Peripheral arterial disease in relation to glycaemic level in an elderly Caucasian population: the Hoorn study. Diabetologia 1995;38:86-96.

9. Young MJ, Boulton AJ, MacLeod AF, et al. A multicentre study of the prevalence of diabetic peripheral neuropathy in the United Kingdom hospital clinic population. Diabetologia 1993;36:150-4.

10. Digital NHS. National Diabetes Inpatient Audit (NaDIA)-2016. 2017. http://www.nice.org.uk/guidance/cg179/resources/guidancepressure-ulcers-prevention-and-management-of-pressure-ulcers (accessed 10 Mar 2017)

11. Pressure ulcers: prevention and management of pressure ulcers [article online]. 2014. http://www.nice.org.uk/guidance/cg179/ resources/guidance-pressure-ulcers-prevention-and-managementof-pressure-ulcers-pdf (accessed 14 Jun 2017).

12. Qaseem A, Mir TP, Starkey M, et al. Risk assessment and prevention of pressure ulcers: a clinical practice guideline from the American College of Physicians. Ann Intern Med 2015;162:359-69.

13. Meng YY, Pickett M, Babey SH, et al. Diabetes tied to a third of California hospital stays, driving health care costs higher. Los Angeles: UCLA Center for Health Policy Research and California Center for Public Health Advocacy, 2014. http://healthpolicy.ucla. edu/publications/search/pages/detail.aspx?PubID=1278 (accessed 14 Jun 2017)

14. Datix Sortware for patient safety. 2017. http://www.datix.co.uk/en/ products/datixweb/incident-reporting (accessed 09 Jun 2017).

15. Classification and Diagnosis of Diabetes. Diabetes Care 2015;38(Supp 1):S8-S16.

16. Rodriguez G, Goldman N. Improved estimation procedures for multilevel models with binary response: a case-study. $J R$ Stat Soc Ser A Stat Soc 2001;165164:339-55.

17. Bannay $\mathrm{A}$, Chaignot $\mathrm{C}$, Blotière $\mathrm{PO}$, et al. The Best Use of the Charlson Comorbidity Index With Electronic Health Care Database to Predict Mortality. Med Care 2016;54:188-94.

18. Delmore B, Lebovits S, Suggs B, et al. Risk factors associated with heel pressure ulcers in hospitalized patients. J Wound Ostomy Continence Nurs 2015;42:242-8.

19. Krishnan S, Nash F, Baker N, et al. Reduction in diabetic amputations over 11 years in a defined U.K. population: benefits of multidisciplinary team work and continuous prospective audit. Diabetes Care 2008;31:99-101.

20. NHS Safety Thermometer. https://www.safetythermometer.nhs.uk/ index.php?option=com_dashboards\&view=classic\&ltemid $=137$ (accessed 14 June 2017).

21. NICE Scope Pressure ulcers: prevention and management of pressure ulcers. https://www.nice.org.uk/guidance/cg179/ documents/pressure-ulcers-scope2 (accessed 10 Aug 2018).

22. Diabetes Facts and Stats. DiabetesUK 2016. https://diabetesresources-production.s3-eu-west-1.amazonaws.com/diabetesstorage/migration/pdf/DiabetesUK_Facts_Stats_Oct16.pdf (accessed 10 Feb 2018).

23. Pancorbo-Hidalgo PL, Garcia-Fernandez FP, Lopez-Medina IM, et al Risk assessment scales for pressure ulcer prevention: a systematic review. J Adv Nurs 2006;54:94-110.

24. Braden BJ, Bergstrom N. Predictive validity of the Braden Scale for pressure sore risk in a nursing home population. Res Nurs Health 1994:17:459-70.

25. Waterlow J. Pressure sores: a risk assessment card. Nurs Times 1985;81:49-55. 